K.S. Toth
Oak Ridge National Laboratory, Oak Ridge, TN 37831

\title{
D.M. Moltz
}

University of South Carolina, Columbia, SC 29208 and

Oak Ridge National Laboratory, Oak Ridge, TN 37831

E.C. Schloemer and M.D. Cable

Lawrence Berkeley Laboratory, Berkeley, CA 94720

F.T. Avignone, III

University of South Carolina, Columbia, SC 29208

Y.A. El lis-Akovali

Oak Ridge National Laboratory, Oak Ridge, TN 37831

\section{SUMMARY}

The present paper discusses mainly the B-delayed proton spectr: of 1470y and of the hitherto unknown isotope, 149 $\mathrm{Er}$. However, following the submittal of the abstract for this conference we have now observed delayed protons following the decay of 145Dy. Additionally, we have identified a 0.5 -s delayed-proton emitter and tentatively assign it to the new isotope, :slyb.

We recently completed an investigation ${ }^{1}$ of $147 \mathrm{~Tb}$ states populated in the decay of 147 Dy. The $s_{1 / 2}, \alpha_{3 / 2}, d_{5 / 2}$, and $g_{7 / 2}$ orbitals were identified. (The $h_{11 / 2}$ state was not observed; however, in a recent note ${ }^{2}$ the $h_{11 / 2}$ and $s_{1 / 2}$ states in $147 \mathrm{~Tb}$ are reported to be at 0 and $50 \mathrm{keV}$, respectively.) With this information and earlier results, the location of these proton orbitals was traced as a function of atomic number in odd- $Z N=82$ nuclei from 135 I to 147 Tb. Their excitation energies varied smoothly with an indication that they may be more tightly bound in $147 \mathrm{~Tb}$, perhaps due to the $Z=64$ subshell (see-e.g. Refs. 3 and 4). To understand more fully the influence of the $Z=64$ gap on the quasiparticle energies, we suggested ${ }^{l}$ that the investigation be extended to 14.9 Ho.

To locate the proton levels in 14910, we searched for the $B$ decay of $149 \mathrm{Fr}$ in a series of $12 \mathrm{C}+144 \mathrm{Sm}$ bombardments made at the Oak Ridge isochronous cyclotron. A helium gas-jet apparatus was used to transport radioactive products to a shielded area suitable for $\gamma$ - and $x$-ray counting. Despite the fact that the new isotope ${ }^{150} \mathrm{Er}$ was identified ${ }^{5}$ and $149 \mathrm{Ho} \gamma$ rays were observed, no transitions could be ascribed to $14^{9} \mathrm{Er}$ decay. Proton spectral measurements ${ }^{6}$ for $A=147$ nuclei have recently attributed a delayed-proton branch to I4? Dy. Because of the energetics involved one would also expect $149 \mathrm{Er}$ to have the same 
mode of decay. In a further effort to identify $149 \mathrm{Er}$, we undertook a search for its $\beta$-delayed-proton decay.

Irradiations were made at the Lawrence Berkeley Laboratory 88-inch cyclotron to take advantage of its higher incident ${ }^{12} \mathrm{C}$ energies. A helium gas-jet apparatus thermalized product recoils and transported them to a collection box for assay with a Si particle telescope and a Ge detector. The telescope, consisting of a $20-\mu m$ $\Delta E$ detector combined with a 300- $m E$ detector, was necessary for the selective detection of low-energy protons in the presence of intense. $B$ radiation and a profusion of $\alpha$ particles emitted in the decay of nearby nuclides. The Ge detector was of the $y-x$ variety, suitable for detecting both low- and high-energy photons. Events registered in each detector were tagged with a time signal for half-life information. Coincidences between particles and $\gamma$ rays were also recorded. To observe protons, however, the ${ }^{12 C}$ beam intensity was maintained at $\sim 1.5 \mu \mathrm{A}$. The Ge detector had to be backed $12 \mathrm{~cm}$ away from the source spot and the greatly reduced geometry resulted in a very low particle-gamma coincidence rate.

Besides ${ }^{144} \mathrm{Sm},{ }^{142} \mathrm{Nd}$ was also irradiated to confirm the existence of $147 \mathrm{Dy}$ delayed-proton activity, to help determine the peak energy for the $(12 \mathrm{C}, 7 \mathrm{n})$ excitation function, and to provide cross-bombardment information. The targets were rare earth oxides enriched in $142 \mathrm{Nd}(97.7 \%)$ and ${ }^{144} \mathrm{Sm}(96.5 \%)$ deposited onto 12.5- m-thick Be foils.

Based on a brief survey run with $142 \mathrm{Nd}$, an incident energy of $\sim 135 \mathrm{MeV}$ was found to produce the maximum yieid for the 14 bym 678.7-keV M4 transition. 1 Figure $1(a)$ shows the proton spectrum accumulated at that bombarding energy following repeated $120-\mathrm{sec}$ irradiation and counting cycles. Subsequent excitation function data established that the proton yield paralleled closely that of the 678.7-keV $\gamma$ ray. Further, a weak group of terbium $k_{\alpha} \times$ rays was observed in coincidence with the protons; thus, $147 \mathrm{Dy}$ is established as the $\beta$-decay precursor. The intensities of a particles and $\gamma$ rays in the well-known $212 \mathrm{pb}$ decay chain were used to normalize the geometries of the $\triangle E-E$ telescope and the $\gamma-x$ detector with respect to one another. The ratio, $I_{\text {protons }} / I_{679}$, was then determined to be $\sim 1.3 \times 10^{-3}$. Because the 678.7-keV $\gamma$ ray is estimated ${ }^{7}$ to be $\lesssim 40 \%$ of the total 147Dy decay strength, the nuclide's delayed-proton branch is $\sim 5 \times 10^{-4}$.

The $A=147$ mass-separated delayed-proton spectrum observed by Klepper et al. ${ }^{6}$ in ${ }^{58} \mathrm{Ni}+92 \mathrm{Mb}$ bombardments is similar to the one shown in Fig. 1(a), particularly with regard to the distinct peaks seen below $4 \mathrm{MeV}$ in excitation energy. Additional measurements mentioned in a "Note Added to Proof" showed that their spectrum was made up of two components: a structureless spectrum extending to $8 \mathrm{MeV}$, assigned to ${ }^{147 \mathrm{Er}}(2.5 \pm 0.2 \mathrm{sec})$, and a spectrum dominated 


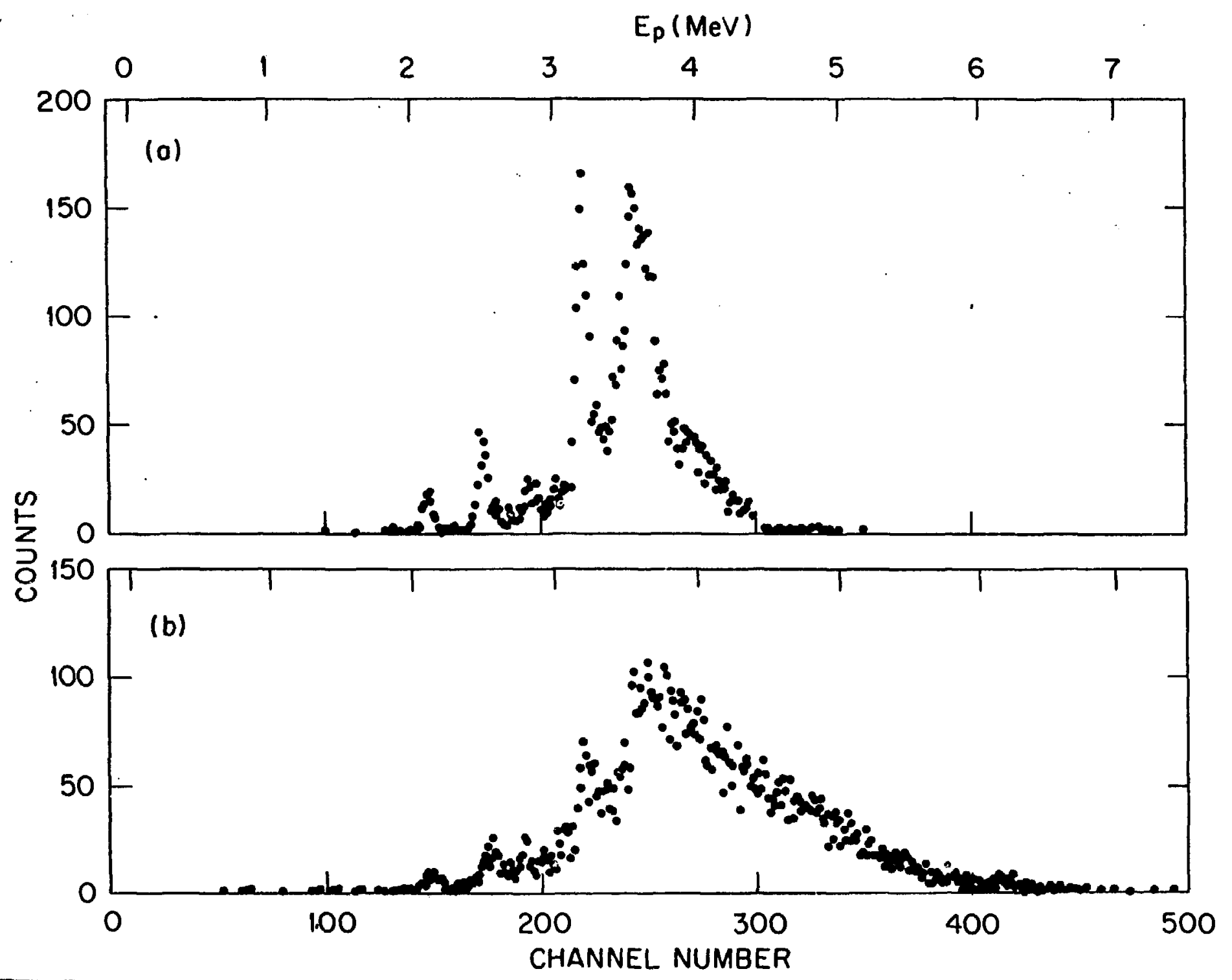

Fig. 1, Delayed-proton spectra observed in ${ }^{12} \mathrm{C}+{ }^{142} \mathrm{Nd}$ [part (a)] and ${ }^{12} \mathrm{C}+144 \mathrm{Sm}$ [part (b)] trradtations made at an incident energy of $\sim 135 \mathrm{MeV}$. 
by sharp peaks, with a cutoff at $\sim 5 \mathrm{MeV}$ and a half-life of $57 \pm 4 \mathrm{sec}$, assigned to $147 \mathrm{Dym}$. These conclusions about the second component agree with our assignment of $147 \mathrm{Dy}$ as the precursor of the delayed-protons in Fig. 1(a).

Figure 1 (b) shows the proton spectrum accumulated in $135-\mathrm{MeV} 12 \mathrm{C}$ irradiations of $144 \mathrm{Sm}$. While the bombardment and counting cycles used to obtain the data were $20 \mathrm{sec}$, preliminary measurements were also done with 5- and 60-sec cycles. Differences and similarities can be immediately perceived between Fig. 1(a) and Fig. 1(b). Most of the peaks seen below $4 \mathrm{MeV}$ in Fig. 1(a) are also seen in Fig. 1(b), though here they are superposed on a much more intense structureless spectrum which extends above $6.5 \mathrm{MeV}$. The indication is that 147Dy produced in the ${ }^{144} \mathrm{Sm}(12 \mathrm{C}, \alpha 5 \mathrm{n})$ reaction is present (the 14 Dym $^{6} 678.7-k e V$ transition is clearly seen in the $\gamma-r$ ay spectra), together with another proton emitter. Figure 2 shows the spectrum that results after subtracting from Fig. 1(b) the contribution due to ${ }^{147} \mathrm{Dy}$ as illustrated by Fig. 1(a). The spectrum in Fig. 2 decays with a half-life of $9 \pm 1 \mathrm{sec}$.

Since this proton emitter was not seen in $12 \mathrm{C}+142 \mathrm{Nd}$ bombardments, it has to be either an erbium or a holmium nuclide. No coincident $K \times$ rays could be observed to establish the atomic number. Neighboring erbium and holmium isotopes have half-lives as follows: $15 \mathrm{u}_{\mathrm{Er},} T_{1 / 2}=20 \pm 2 \mathrm{sec}$ (Ref. 5); $150 \mathrm{Hb}$, $T_{1 / 2}=26 \pm 2 \mathrm{sec}$ and $90 \pm 20 \sec (\operatorname{Ref} .5) ; 14 \% 0, T_{1 / 2}=21 \pm 2 \mathrm{sec}(\operatorname{Ref} .8)$; $148 \mathrm{Er}, T_{1 / 2}=4.5 \pm 0.4 \mathrm{sec}$ (Ref. 9); and $14 \% \mathrm{Ho}, T_{1 / 2}=9 \pm 2 \mathrm{sec}$ (Ref. 8). Although 148 Ho has a $9-5 e c$ half-1ife, its $\gamma$ rays (Ref. 8 ) were not observed below $135 \mathrm{MeV}$ and increased in intensity up to our maximum bombarding energy of $155 \mathrm{MeV}$. Such a variation with incident energy is inconsistent with that seen for the delayed protions; their yield as a function of energy was consistent with an $A=149$ product. Therefore, we assign the $9-5 e c$ activity to the $\beta$ decay of the hitherto unidentified isotope, $149 \mathrm{Er}$.

Next we expanded our study by searching for the delayed-proton branch of 145Dy, an isotope whose half-life has been reported to be $18 \pm 3 \mathrm{sec}$ (Ref. 10) and $13.6 \pm 1.0 \mathrm{sec}$ (Ref. 9). In a series of ${ }^{12} \mathrm{C}+142 \mathrm{Nd}$ bombardments (from 135 to $195 \mathrm{MeV}$ ) yields were measured for $\gamma$ rays belonging to $146 \mathrm{by}$ (Refs. 9 and 11), ${ }^{146} \mathrm{~Tb}$ (Ref. 12), ${ }^{145 \mathrm{Dy}}$ (Refs. 9 and 10), 145 Tb (Refs. 9 and 13), and $144 \mathrm{~Tb}$ (Refs. 9 and 13). An energy of $\sim 188 \mathrm{MeV}$ was then selected to emphasize the $142 \mathrm{Nd}\left({ }^{12} \mathrm{C}, 9 \mathrm{n}\right)$ reaction. Figure 3 shows the accumulated delayed-proton spectrum. We assign these $8-d e l$ ayed protons to $1450 \mathrm{y}$ on the basis of excitation function data and on the fact that they decayed with a (15 \pm 4$)-\sec$ half-life.

Further, in an attempt to identify $15 \mathrm{lyb}$, proton and $\gamma$-ray spectra were investigated in $160+144 \mathrm{Sm}$ irradiations. Yields as a function of incident 


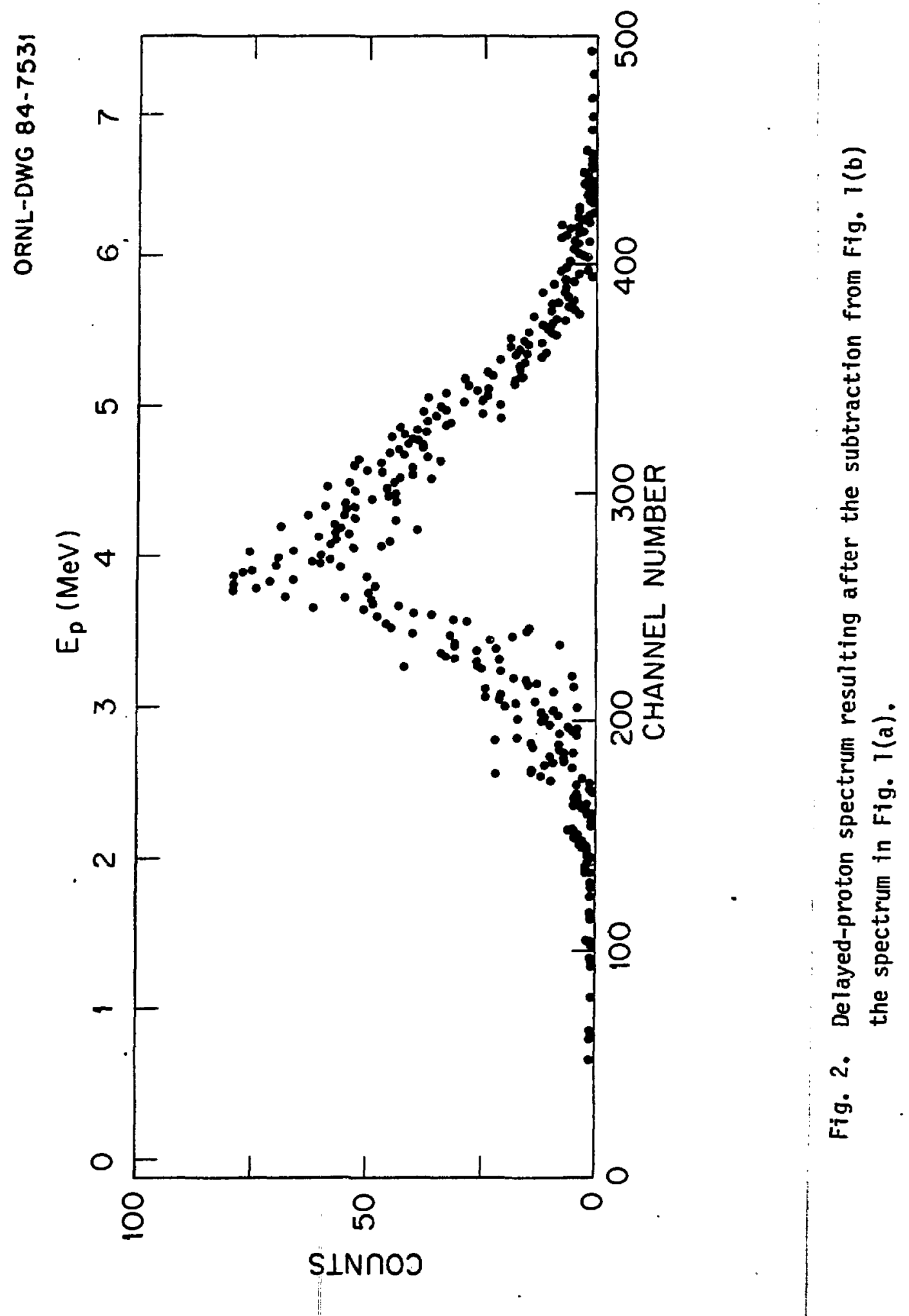




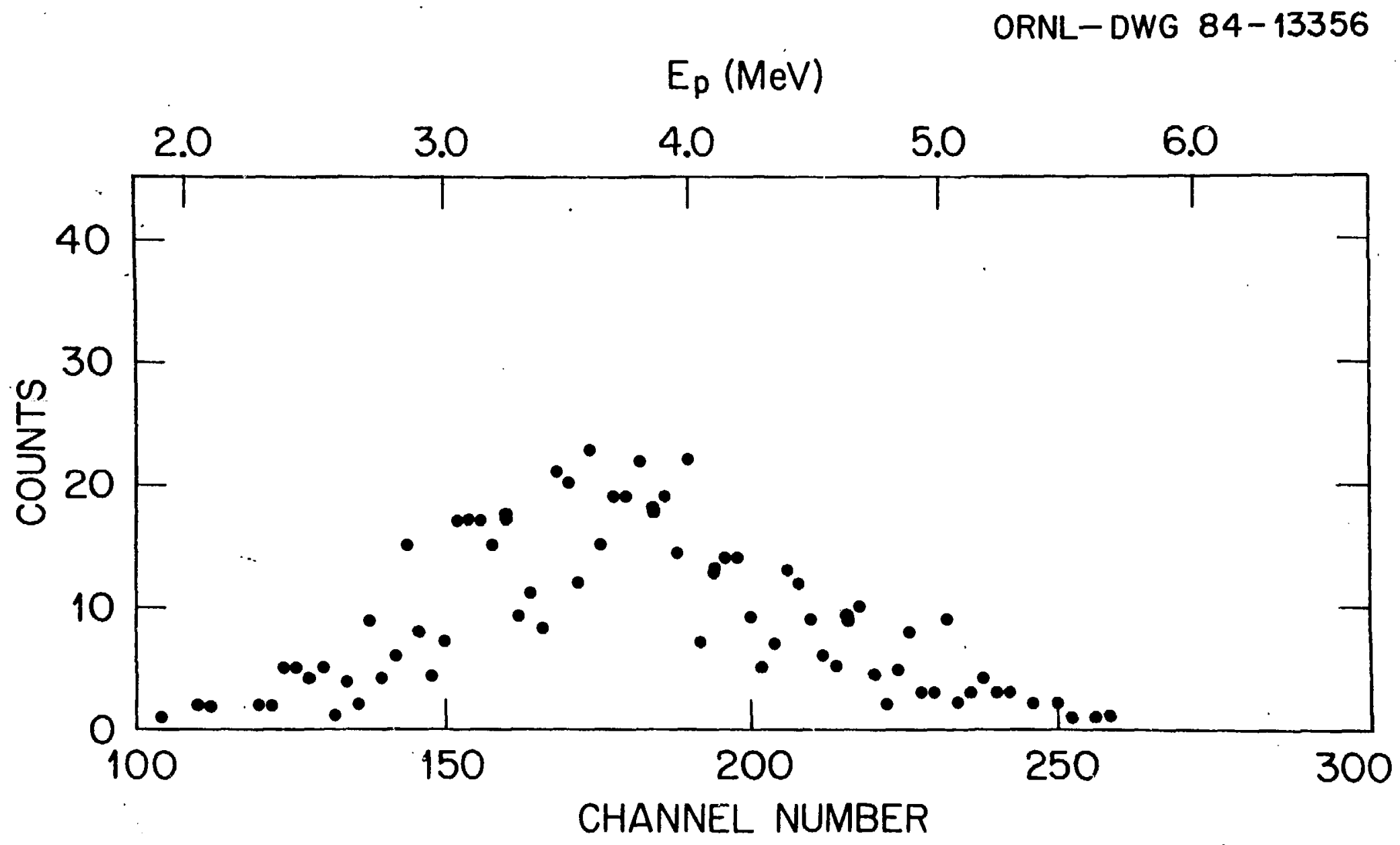

Fig, 3, Delayed-proton spectrum observed in $188-\mathrm{MeV}{ }^{12} \mathrm{C}$ bombardments of ${ }^{142} \mathrm{Nd}$. 
energy for $\gamma$ rays known ${ }^{14}$ to follow the $B$ decays of $152 \mathrm{Yb},{ }^{152} \mathrm{Tm}$, and $151 \mathrm{Tm}$ were used to select the peak of the $(160,9 n)$ excitation function. Preliminary analyses indicate the existence of a delayed-proton emitter with a half-1ife of $\sim 0.5$ sec which we tentatively assign to the new isotope $151 \mathrm{Yb}$.

The intrinsic structure of delayed-proton spectra that accompany heavy mass precursors usually is not resolved due to the large density of states in the excitation energy range fed by the $B$ decay. In Fig. 1(a), however, the peaks have full widths at half-maximum which are on the order of the $\triangle E-E$ detector resolution, i.e., $\sim 60 \mathrm{keV}$. The indication is that the $147 \mathrm{Dy} \beta$ decay is sampling either selected $147 \mathrm{~Tb}$ states or else an energy region in $147 \mathrm{~Tb}$ where the level density is not high. The reader is reminded that $147 \mathrm{~Tb}$ consists of a single proton coupled to the doubly-closed core of $146 \mathrm{Gd}(N=82$ and $Z=64)$. In Fig. 2, while there are peaks in the lower half of the spectrum, the structure is much less distinct than in Fig. 1(a). This could be due to a larger level density in 149\%, a nucleus still with 82 neutrons but now having three protons beyond $z=64$. Finally, there is no indication of peaks in Fig. 3 ; here the $B$ decay is sampling levels in a nucleus, $145 \mathrm{~Tb}$, with no shell closures.

High-energy endpoints of delayed-proton spectra are fixed by differences between the electron-capture (EC) decay energies of the parents and the proton binding energies in the corresponding EC daughters. Predicted $Q_{E C}-B_{p}$ values from the 1977 Atomic Mass Evaluation 15 for 1450y, 147Dy, and $14 \mathrm{~g}^{\mathrm{Er}}$ are 5.70, 4.42, and 6.44 MeV, respectively. (Note, EC decay energies for $1450 \mathrm{y}$ and $14 \mathrm{~g}^{\mathrm{Er}}$ are not listed in Ref. 15; based on decay energies for nearby isotopes we estimated the two $Q_{E C}$ values to be 7.24 and $7.50 \mathrm{MeV}$, respectively.) These predictions are consistent with our data [see Figs. 1(a), 2, and 3].

Oak Ridge National Laboratory is operated by Martin Marietta Energy Systems, Inc. for the U.S. Department of Energy under Contract No. DE-AC05-840R21400. Work at Lawrence Berkeley Laboratory is done under Contract No. DE-AC03-76SF00098 with the U.S. Department of Energy. Support was also provided by the U.S. Department of Energy under Contract No. DE-ASO9-79ERI0434 with the University of South Carolina. References

1. K.S. Toth et a 1., Phys. Rev. C 25, 667 (1982).

2. G.D. Alkhazov et al., Z. Phys. A310, 247 (1983).

3. K.S. Toth et a T., Phys. Rev. C 2, 1480 (1970).

4. W.-D. Schmidt-0tt and K.S. Toth, Phys. Rev. C 13, 2574 (1976).

5. D.M. Moltz et al., Phys. Rev. C 26,1316 (1982).

6. 0. Klepper et aT., Z. Phys. A305, 125 (1982).

7. A.E. Rainis, K.S.' Toth and C.R. Bingham, Phys. Rev. C 13, 1609 (1976).

8. K.S. Toth et al., Phys. Rev. C 19,482 (1979).

9. E. Nolte et a t., Z. Phys. A306, 223 (1982).

10. G.D. Alkhazov et a 1., Z. Phys. A305, 185 (1982).

11. G.D. Alkhazov et at., Acta Phys. Polonica B12, 825 (1981).

12. K.S. Toth, Phys. Rev. C 22, 1341 (1980).

13. D.C. Sousa et al., Phys. Rev. C 25, 1012 (1982).

14. E. Nolte et al., Z. Phys. A309, $\frac{33}{33}$ (1982).

15. A.H. Wapstra and K. Bos, At. Data Nucl. Data Tables 19, 175 (1977). 


\section{DISCLAIMER}

This report was prepared as an account of work sponsored by an agency of the United States Government. Neither the United States Government nor any agency thereof, nor any of their employees, makes any warranty, express or implied, or assumes any legal liability or responsibility for the accuracy, completeness, or usefulness of any information, apparatus, product, or process disclosed, or represents that its use would not infringe privately owned rights. Reference herein to any specific commercial product, process, or service by trade name, trademark, manufacturer, or otherwise does not necessarily constitute or imply its endorsement, recommendation, or favoring by the United States Government or any agency thereof. The views and opinions of authors expressed herein do not necessarily state or reflect those of the United States Government or any agency thereof. 